\title{
Generalized Sampling: Stability and Performance Analysis
}

\author{
Michael Unser, Senior Member, IEEE, and Josiane Zerubia, Member, IEEE
}

\begin{abstract}
Generalized sampling provides a general mechanism for recovering an unknown input function $f(x) \in \mathcal{H}$ from the samples of the responses of $m$ linear shift-invariant systems sampled at $1 / m$ th the reconstruction rate. The system can be designed to perform a projection of $f(x)$ onto the reconstruction subspace $V(\varphi)=\operatorname{span}\{\varphi(x-k)\}_{k \in Z}$; for example, the family of bandlimited signals with $\varphi(x)=\operatorname{sinc}(x)$. This implies that the reconstruction will be perfect when the input signal is included in $V(\varphi)$ : the traditional framework of Papoulis' generalized sampling theory. Otherwise, one recovers a signal approximation $\tilde{f}(x) \in V(\varphi)$ that is consistent with $f(x)$ in the sense that it produces the same measurements. To characterize the stability of the algorithm, we prove that the dual synthesis functions that appear in the generalized sampling reconstruction formula constitute a Riesz basis of $V(\varphi)$, and we use the corresponding Riesz bounds to define the condition number of the system. We then use these results to analyze the stability of various instances of interlaced and derivative sampling. Next, we consider the issue of performance, which becomes pertinent once we have extended the applicability of the method to arbitrary input functions, that is, when $\mathcal{H}$ is considerably larger than $V(\varphi)$, and the reconstruction is no longer exact. By deriving general error bounds for projectors, we are able to show that the generalized sampling solution is essentially equivalent to the optimal minimum error approximation (orthogonal projection), which is generally not accessible. We then perform a detailed analysis for the case in which the analysis filters are in $L_{2}$ and determine all relevant bound constants explicitly. Finally, we use an interlaced sampling example to illustrate these various calculations.
\end{abstract}

Index Terms - Error analysis, projection operators, restoration, sampling, splines, wavelets.

\section{INTRODUCTION}

$\mathbf{T}$ HE GENERALIZED sampling problem is to reconstruct an unknown continuously defined input function $f(x) \in$ $\mathcal{H}$ from the samples of the responses of $m$ linear shift-invariant systems sampled at $1 / m$ th the reconstruction rate. Papoulis provided an elegant solution for the particular case where the Hilbert space $\mathcal{H}$ is the class of bandlimited functions [10]. A typical example (derivative sampling) is to sample both the signal and its derivative at half the Nyquist rate. Recently, we have extended the scope of Papoulis' theory by introducing a formal distinction between the input space $\mathcal{H}$ and the reconstruction space, which we denote by $V(\varphi)$ [18]. The idea is to allow $\mathcal{H}$ to be considerably larger than $V(\varphi)$-typically,

Manuscript received January 13, 1997; revised April 25, 1997. The associate editor coordinating the review of this paper and approving it for publication was Dr. Xiang-Gen Xia.

M. Unser is with the Department of Micro-Engineering, Swiss Federal Institute of Technology, Lausanne, $\mathrm{CH}-1015$ Switzerland.

J. Zerubia is with INRIA, F-06902 Sophia Antipolis, France.

Publisher Item Identifier S 1053-587X(97)07334-0.
$\mathcal{H}$ is the space $L_{2}$ of finite energy signals, and $V(\varphi)$ is the subspace of bandlimited functions generated from the integer translates of $\varphi(x)=\operatorname{sinc}(x)$. We have shown that one could still obtain a meaningful reconstruction when the input function $f(x)$ is arbitrary and not necessarily bandlimited; the reconstruction $\tilde{f}(x) \in V(\varphi)$ is not exact but is a consistent approximation of the unknown input signal in the sense that it produces exactly the same measurements. In other words, the system is designed to perform a projection from $\mathcal{H}$ onto $V(\varphi)$. One of the primary implications is that the reconstruction is exact when $f(x) \in V(\varphi)$, which corresponds to the narrower framework of traditional sampling theories (Shannon and Papoulis). The advantage of this recent shift of paradigm is that the theory becomes more realistic; there is no artificial restriction on the class of input functions. Our generalization also allows for other nonbandlimited reconstruction models such as splines and wavelets [2], [7], [16], [19]. Although this theory requires a certain degree of abstraction for a rigorous specification of the various signal spaces, its end result is a simple reconstruction algorithm that takes the familiar form of the multivariate deconvolution filter (cf., [18, Section III-C]).

In principle, a consistent signal recovery is possible for almost any set of analysis functions provided that some invertibility condition is met (cf., Sect. II-C). Unfortunately, the method will not always yield a reconstruction algorithm that is stable numerically. This fact had already been pointed out by Marks and Cheung, who identified special instances of derivative sampling where the reconstruction is ill-posed [4], [9]. The first objective of this paper is to investigate this stability issue within our more general framework and derive figures of merit that characterize the overall behavior of the algorithm. The second objective is to get a better handle on the issue of performance, which is a new problem raised by our less restrictive formulation. Indeed, the essential difference with the conventional formulation is that the reconstruction is no longer exact unless the input signal is already included within the reconstruction space. Ideally, we would like to recover the minimum error approximation of $f(x)$ in $V(\varphi)$, which corresponds to the orthogonal projection of $f(x)$ onto $V(\varphi)$. Unfortunately, this optimal solution is generally nonrealizable unless we have additional information available. We will show, however, that the present algorithm provides an estimate that is essentially equivalent to the minimum error solution.

The paper is organized as follows. In Section II, we give a brief axiomatic formulation of our generalized sampling theorem (Theorem 1). In particular, we discuss our three basic assumptions a1)-a3) that ensure that the reconstruction 
problem is well-defined mathematically. In Section III, we prove that the dual reconstruction functions in Theorem 1 constitute a Riesz basis of $V(\varphi)$ (Theorem 2). This result turns out to be very relevant to the stability issue because the corresponding Riesz bounds provide us with the condition number of the algorithm. We then present specific examples of stability analyzes (interlaced and derivative sampling) to illustrate this aspect of the theory. In Section IV, we turn to the issue of performance. First, we investigate the problem at an abstract level, deriving general error inequalities for projection operators. Our key motivation is to relate the present approximation error $\|f-\tilde{f}\|$ to that of the least squares solution (Theorem 3). We show that the degree of optimality of our algorithm depends on the norm of the underlying projection operator $\tilde{P}$ (Proposition 1), which is a quantity that is itself related to an abstract angle between two subspaces (Theorem 4). In Section IV-C, we get back to our sampling problem and derive all relevant bound constants for the special case where the analysis functions are in $L_{2}$. In particular, we obtain an explicit formula for the constant $C_{\phi}$ that appears in Condition $\left(\mathrm{a} 1^{\prime}\right)$ (Proposition 2). Finally, in Section IV-D, we consider an example of interlaced sampling with prefiltering to illustrate these various calculations. We show that the prefiltering step prior to sampling is beneficial for performance and can even compensate for the fact that the sampling is nonuniform.

\section{GenERALIZED SAMPLING}

In this section, we briefly review the generalized sampling theory that was developed in [18]. The corresponding system is schematically represented in Fig. 1. The continuous-time signal $f(x)$ is convolved with a bank of analysis filters $h_{i}(x)=$ $\phi_{i}(-x), i=1, \cdots, m$, the responses of which are sampled at $1 / m$ th the reconstruction rate to yield the measurement vector $\mathbf{g}_{m}(k)=\left[g_{1}(m k), g_{2}(m k), \cdots, g_{m}(m k)\right]$. Our main reason for introducing the time-reversed analysis functions $\phi_{i}(x)=h_{i}(-x)$ is that we can describe the measurement process in terms of inner products

$$
\begin{aligned}
g_{i}(m k) & =\left(h_{i} * f\right)(m k) \\
& =\left\langle f(x), \phi_{i}(x-m k)\right\rangle
\end{aligned}
$$

which facilitates the mathematical analysis. These discrete measurements are then combined to produce the continuoustime output $\tilde{f}(x)$. The system is essentially the same as the one considered by Papoulis, except that the output $\tilde{f} \in V(\varphi)$ is only an approximation of the input $f \in \mathcal{H}$, where $\mathcal{H}$ is a class of functions that is considerably larger than $V(\varphi)$.

The reconstruction system works for almost any set of analysis filters, provided that some invertibility condition [cf., a3) below] is met. Before restating our generalized sampling theorem, we first review the underlying mathematical assumptions that will play a crucial role in all subsequent derivations.

The mathematical notations are the same as those used in [18]. In particular, we use a "hat" symbol to denote the $z$ transform of a scalar or a matrix sequence, i.e., $\hat{a}(z)=\sum_{k} a(k) z^{-k}$ and $\hat{\mathbf{A}}(z)=\sum_{k} \mathbf{A}(k) z^{-k}$. The Fourier transform is obtained by setting $z=e^{j \omega}$.

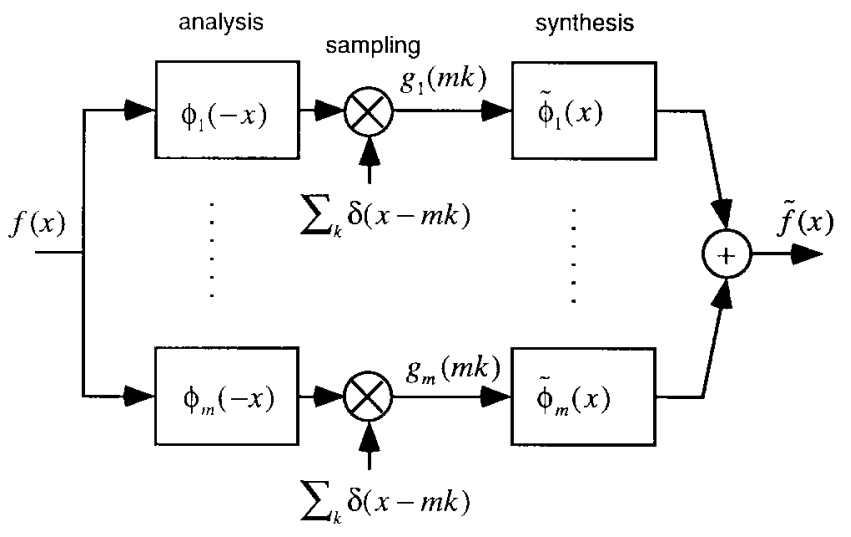

Fig. 1. Generalized sampling: The problem is to recover an unknown function $f(x) \in \mathcal{H}$ from its discrete measurements. These measurements are obtained by sampling the output of a $m$ channel analysis filterbank. The reconstruction process involves the dual synthesis functions $\phi_{i}(x)$ in Theorem 1; it yields a signal approximation $\tilde{f}(x) \in V(\varphi)$. In general, the reconstruction is not perfect because the input space $\mathcal{H}$ is much richer than the reconstruction subspace $V(\varphi)$.

\section{A. Extended Class of Input Functions}

In principle, our results are applicable for any input function $f(x)$, provided that its sequence of measurements is well defined in the $l_{2}$ sense. In the companion paper [18], we have considered the most general case where the class of admissible input functions $\mathcal{H}$ is such that

Condition a1):

$$
\forall f \in \mathcal{H}, \quad \sum_{i=1}^{m} \sum_{k \in Z}\left|\left\langle f(x), \phi_{i}(x-m k)\right\rangle\right|^{2}<+\infty .
$$

For some of our results here, we will need a stronger specification of the input space and require that there exists a finite constant $C_{\phi}$ such that

$$
\text { Condition } \left.\mathrm{a}^{\prime}\right): \quad \forall f \in \mathcal{H}, \quad\left\|\mathbf{g}_{m}\right\|_{l_{2}} \leq C_{\phi} \cdot\|f\|_{L_{2}} .
$$

Note that $\mathrm{a} 1^{\prime}$ ) implies a1), whereas the converse is not necessarily true. However, in Section IV-C, we will show that if the $\phi_{i}$ 's are in $L_{2}$, then it is usually possible to consider any possible finite energy input function (i.e., $\mathcal{H}=L_{2}$ ) and determine the constant $C_{\phi}$ explicitly. In other words, there is virtually no constraint on the input of the system. For more idealized cases where the analysis functions are distributions, we need to be more restrictive and specify the largest admissible input space $\mathcal{H}$ on a case-by-case basis. A typical example is when the $\phi_{i}$ 's are Dirac-delta functions (interlaced sampling). For this particular case, it can be shown that Condition a1) is satisfied if $\mathcal{H}=W_{2}^{1}$, where $W_{2}^{1}$ is Sobolev space of order 1 . Note that this is a very mild constraint because we are only requiring that the input function $f$ and its derivative $f^{(1)}$ are both in $L_{2}$. If we want Condition $\left.\mathrm{a} 1^{\prime}\right)$ to be satisfied as well, we can consider the subclass of functions whose generalized bandwidth $\|\omega \hat{f}\|_{L_{2}} /\|\hat{f}\|_{L_{2}}$ is bounded. These functions are predominantly lowpass but not necessarily bandlimited. This is also equivalent to defining $\mathcal{H}_{\gamma}=\left\{f:\left\|f^{(1)}\right\|_{L_{2}} \leq \gamma \cdot\|f\|_{L_{2}}<+\infty\right\}$, where $\gamma$ is an appropriate bandwidth parameter; this subspace is smaller than $W_{2}^{1}$ but is still much larger than any reconstruction space that 
we may choose. At any rate, our main point is that we can easily select an admissible input space $\mathcal{H}$ that is considerably larger than the family of bandlimited function considered in Shannon's or Papoulis' sampling theories.

\section{B. Reconstruction Subspaces}

The reconstructed signal $\tilde{f}$, on the other hand, belongs to the much smaller reconstruction space

$$
V(\varphi)=\left\{\tilde{f}(x)=\sum_{k \in Z} c(k) \varphi(x-k) \mid c(k) \in l_{2}\right\}
$$

where $\varphi(x)$ is a given generating function. This covers the bandlimited case with $\varphi(x)=\operatorname{sinc}(x)$ but also other more recent signal representation models such as splines and wavelets [7], [19]. We require that $V(\varphi)$ is a well-defined (closed) subspace of $L_{2}$. This is equivalent to the condition (cf., [2])

$$
\text { Condition a2): } 0<A_{\varphi} \leq \hat{a}_{\varphi}\left(e^{j \omega}\right) \leq B_{\varphi}<+\infty \text { a.e. }
$$

where the central inequality holds almost everywhere. The constants $A_{\varphi}$ and $B_{\varphi}$ are the lower and upper Riesz bounds, respectively, whereas $\hat{a}_{\varphi}\left(e^{j \omega}\right)$ is the Fourier transform of the autocorrelation sequence $a_{\varphi}(k)$, which is defined as $a_{\varphi}(k):=$ $\langle\varphi(x-k), \varphi(x)\rangle$.

\section{Invertibility Condition}

Our solution involves a multivariate reconstruction filter $Q$, which is specified via a matrix inversion in the $z$-transform domain

$$
\hat{\mathbf{Q}}(z)=\hat{\mathbf{A}}_{\phi \varphi}^{-1}(z)
$$

where $\hat{\mathbf{A}}_{\phi \varphi}(z)$ is the $z$-transform of the input-output crosscorrelation matrix sequence $\mathbf{A}_{\phi \varphi}(k)$ whose scalar entries are given by

$$
\left[\mathbf{A}_{\phi \varphi}\right]_{i, j}(k)=\left(h_{i} * \varphi\right)(m k-j+1) .
$$

Note that $\hat{\mathbf{A}}_{\phi \varphi}(z)=\hat{\mathbf{A}}_{\text {poly }}(z)$ can also be interpreted as the polyphase matrix of the auxiliary analysis filterbank $a_{i}(k)=$ $\left(h_{i} * \varphi\right)(k), i=1, \cdots, m$ (cf., [18, Section V]). We require the filter $Q$ and its inverse to be stable in the sense that there exist two constants $m_{Q}$ and $M_{Q}$ such that

Condition a3):

$$
\left\{\begin{array}{l}
m_{Q}^{2}=\underset{\omega \in[0,2 \pi)}{\operatorname{ess} \inf _{\min }\left[\hat{\mathbf{Q}}^{T}\left(e^{-j \omega}\right) \cdot \hat{\mathbf{Q}}\left(e^{j \omega}\right)\right]>0} \\
M_{Q}^{2}=\underset{\omega \in[0,2 \pi)}{\operatorname{ess} \sup } \lambda_{\max }\left[\hat{\mathbf{Q}}^{T}\left(e^{-j \omega}\right) \cdot \hat{\mathbf{Q}}\left(e^{j \omega}\right)<+\infty\right.
\end{array}\right.
$$

where $\lambda_{\min }[\cdot]$ and $\lambda_{\max }[\cdot]$ denote the smallest and largest eigenvalue of the positive definite matrix argument, respectively. Condition a3) makes use of the essential infimum and essential supremum operators, which yield bounds that are valid almost everywhere. Note that we could also have expressed these constants in terms of the maximum and minimum eigenvalues of $\left[\hat{\mathbf{A}}_{\phi \varphi}^{T}\left(e^{-j \omega}\right) \cdot \hat{\mathbf{A}}_{\phi \varphi}\left(e^{j \omega}\right)\right]$.

\section{Generalized Sampling Theorem}

Theorem 1: Let $f(x) \in \mathcal{H}$ be the unknown input function in Fig. 1. Under Assumptions a1)-a3), there is a unique signal approximation $\tilde{f}(x) \in V(\varphi)$ that is consistent with $f(x)$ in the sense that

$$
\forall f \in \mathcal{H}, \quad\left\langle\tilde{f}(x), \phi_{i}(x-m k)\right\rangle=\left\langle f(x), \phi_{i}(x-m k)\right\rangle .
$$

This approximation always exits and is given by

$$
\tilde{f}(x)=\sum_{i=1}^{m} \sum_{k \in Z} g_{i}(m k) \tilde{\phi}_{i}(x-m k)=\tilde{P} f(x)
$$

and the underlying operator $\tilde{P}$ is a projector from $\mathcal{H}$ into $V(\varphi)$. The synthesis functions $\tilde{\phi}_{i}$ are given by

$$
\tilde{\phi}_{i}(x)=\sum_{k \in Z} q_{i}(k) \varphi(x-k), \quad(i=1, \cdots, m)
$$

where the sequences $q_{i}(k)$ are determined as

$$
\left[\begin{array}{lll}
\hat{q}_{1}(z) & \cdots & \hat{q}_{m}(z)
\end{array}\right]=\left[\begin{array}{lll}
1 & \cdots & z^{-m+1}
\end{array}\right] \cdot \hat{\mathbf{Q}}\left(z^{m}\right) ;
$$

where the filter matrix $\hat{\mathbf{Q}}(z)$ is specified by (3).

The important point here is that we can produce a signal approximation that is consistent with the unknown input $f(x)$ in the sense that it would produce exactly the same measurements if it was reinjected into the system. In this sense, $\tilde{f}(x)$ and $f(x)$ are essentially equivalent because they "look" the same for the end user who can only observe $f(x)$ indirectly through its measurements. For the proof and a detailed interpretation of this result, see [18]. This earlier report also contains a number of illustrative examples, some of which will be revisited in the next section. The above theorem is compatible with Papoulis's generalized sampling expansion in [10], which corresponds to the more restrictive case $\mathcal{H}=V$ (sinc).

\section{STABility ANALYSIS}

To simplify the analysis, we will adopt a more compact vector notation. First, we introduce the $m$-vector representation of the generating function $\varphi$

$$
\boldsymbol{\Psi}(x)=\left[\begin{array}{c}
\varphi(x) \\
\varphi(x-1) \\
\vdots \\
\varphi(x-m+1)
\end{array}\right] .
$$

Combining this definition with (7) and (8), we obtain another equivalent expression for the dual synthesis functions in Theorem 1

$$
\tilde{\boldsymbol{\Phi}}(x)=\sum_{k \in Z} \mathbf{Q}^{T}(k) \mathbf{\Psi}(x-m k)
$$

where $\tilde{\boldsymbol{\Phi}}(x)=\left[\tilde{\phi}_{1}(x), \cdots, \tilde{\phi}_{m}(x)\right]$ is the corresponding synthesis vector. Having introduced this notation, we can represent the reconstructed signal in (6) in the more compact form

$$
\tilde{f}(x)=\sum_{k \in Z} \mathbf{g}_{m}^{T}(k) \tilde{\mathbf{\Phi}}(x-m k)
$$


where $\mathbf{g}_{m}(k)$ is the measurement vector collected in Fig. 1. Interestingly, the synthesis functions $\tilde{\boldsymbol{\Phi}}(x)$ have the following property.

Theorem 2: The set $\{\tilde{\boldsymbol{\Phi}}(x-m k)\}_{k \in Z}$ constitutes a Riesz basis of $V(\varphi)$. In other words, $\forall \tilde{f}(x) \in V(\varphi)$, there exists a sequence $\mathbf{g}(k) \in l_{2}^{m}$ and two strictly positive constants $A$ and $B$ such that

$$
\begin{aligned}
& \text { (i) } \tilde{f}(x)=\sum_{k \in Z} \mathbf{g}^{T}(k) \tilde{\boldsymbol{\Phi}}(x-m k) \\
& \text { (ii) } \quad A \cdot\|\mathbf{g}\|_{l_{2}}^{2} \leq\|\tilde{f}(x)\|_{L_{2}}^{2} \leq B \cdot\|\mathbf{g}\|_{l_{2}}^{2} .
\end{aligned}
$$

Equation (12) expresses the fact that the functions $\tilde{\phi}_{i}$ span the space $V(\varphi)$. Equation (13) is the standard definition of a Riesz basis. In particular, it ensures that the $\tilde{\phi}_{i}$ 's are linearly independent. Going back to the generalized sampling expansion (6), this inequality also provides crucial information about the stability of the algorithm. Specifically, if we consider a perturbation $\Delta \mathrm{g}$ on the input, by linearity, we have that

$$
\sqrt{A} \leq \frac{\|\Delta \tilde{f}(x)\|_{L_{2}}}{\|\Delta \mathbf{g}\|_{l_{2}}} \leq \sqrt{B}
$$

where $\Delta \tilde{f}$ is the corresponding variation on the output. Combining this relation with (13), we can also show that

$$
\begin{aligned}
\sqrt{\frac{A}{B}}\left(\frac{\|\Delta \mathbf{g}\|_{l_{2}}}{\|\mathbf{g}\|_{l_{2}}}\right) & \leq\left(\frac{\|\Delta \tilde{f}(x)\|_{L_{2}}}{\|\tilde{f}(x)\|_{L_{2}}}\right) \\
& \leq \sqrt{\frac{B}{A}}\left(\frac{\|\Delta \mathbf{g}\|_{l_{2}}}{\|\mathbf{g}\|_{l_{2}}}\right) .
\end{aligned}
$$

Based on this inequality, we define the condition number of the system given by the ratio

$$
\alpha_{\tilde{\phi}}=\sqrt{\frac{B_{\tilde{\phi}}}{A_{\tilde{\phi}}}} \geq 1
$$

where $A_{\tilde{\phi}}$ and $B_{\tilde{\phi}}$ are tightest possible bound constants. This quantity provides an estimate of the relative error propagation in the system; it is a good indicator of the overall robustness of the algorithm. The most favorable situation is obviously $\alpha_{\tilde{\phi}}=1$, in which case the dual basis is also orthogonal.

Since $V(\varphi)=\operatorname{span}\{\boldsymbol{\Phi}(x-m k)\}_{k \in Z}$, we can use Aldroubi's Theorem (see Theorem 5 in Section IV-C) to obtain the relevant bound constants explicitly

$$
\left\{\begin{array}{l}
A_{\tilde{\phi}}=\underset{\omega \in[0,2 \pi)}{\operatorname{ess} \inf } \lambda_{\min }\left[\hat{\mathbf{A}}_{\tilde{\phi}}\left(e^{j \omega}\right)\right] \\
B_{\tilde{\phi}}=\underset{\omega \in[0,2 \pi)}{\operatorname{ess} \sup } \lambda_{\max }\left[\hat{\mathbf{A}}_{\tilde{\phi}}\left(e^{j \omega}\right)\right]
\end{array}\right.
$$

where the $m \times m$ matrix $\hat{\mathbf{A}}_{\tilde{\phi}}(z)$ is the $z$-transform of the autocorrelation matrix sequence $\mathbf{A}_{\tilde{\phi}}(k)=\left\langle\tilde{\boldsymbol{\Phi}}(x-m k), \tilde{\boldsymbol{\Phi}}^{T}(x)\right\rangle$. To determine these bounds numerically, we still need to characterize the matrix $\hat{\mathbf{A}}_{\tilde{\phi}}\left(e^{j \omega}\right)$. Using (10), we derive the relation

$$
\hat{\mathbf{A}}_{\tilde{\phi}}(z)=\hat{\mathbf{Q}}^{T}\left(z^{-1}\right) \cdot \hat{\mathbf{A}}_{\varphi}(z) \cdot \hat{\mathbf{Q}}(z)
$$

which involves the transfer function $\hat{\mathbf{Q}}(z)$ of the multivariate deconvolution filter $Q$ as well as $\hat{\mathbf{A}}_{\varphi}(z)$, the $z$-transform of the $m \times m$ autocorrelation matrix sequence

$$
\mathbf{A}_{\varphi}(k)=\left\langle\boldsymbol{\Psi}(x-m k), \boldsymbol{\Psi}^{T}(x)\right\rangle .
$$

By construction, $\mathbf{A}_{\varphi}(k)$ has a special cyclic structure. Its entries are given by

$$
\begin{aligned}
{\left[\mathbf{A}_{\varphi}\right]_{i, j}(k) } & =\langle\varphi(x-i+1-m k), \varphi(x-j+1)\rangle \\
& =a_{\varphi}(m k+i-j) .
\end{aligned}
$$

where $a_{\varphi}(k)$ is the univariate autocorrelation sequence defined in Section II-B. Using the standard rules of decimation, we obtain the corresponding matrix entries in the Fourier domain

$$
\left[\hat{\mathbf{A}}_{\varphi}\right]_{i, j}\left(e^{j \omega}\right)=\frac{1}{m} \sum_{k=0}^{m-1} \hat{a}_{\varphi}\left(\frac{\omega+2 k \pi}{m}\right) e^{j[(\omega+2 k \pi) / m](i-j)} .
$$

We can then apply the same technique as in [5, Section III-B] to show that $\hat{\mathbf{A}}_{\varphi}\left(e^{j \omega}\right)$ is diagonalized by an $m \times m$ unitary Fourier transform matrix and that its eigenvalues are given by

$$
\lambda_{k}\left[\hat{\mathbf{A}}_{\varphi}\left(e^{j \omega}\right)\right]=\hat{a}_{\varphi}\left(\frac{\omega+2 k \pi}{m}\right), \quad k=0, \cdots, m-1
$$

which establishes the connection with the univariate spectral characterization in Section II-B.

\section{A. Proof of Theorem 2}

We start with the manipulation

$$
\begin{aligned}
& \sum_{l \in Z} \mathbf{A}_{\phi \varphi}^{T}(l) \tilde{\boldsymbol{\Phi}}(x-m l-m k) \\
& \quad=\sum_{l \in Z}\left(\mathbf{A}_{\phi \varphi}^{T} * \mathbf{Q}^{T}\right)(l) \mathbf{\Psi}(x-m l-m k) \\
& =\mathbf{\Psi}(x-m k)
\end{aligned}
$$

where we have used (10) and the fact that the reconstruction filter $\mathbf{Q}(k)$ is the convolution inverse of the matrix sequence $\mathbf{A}_{\phi \varphi}(k)$. Since (22) holds for any integer $k$, we have effectively expressed the basis functions of $V(\varphi)$ in terms of the $\tilde{\phi}_{i}$ 's, which proves that the representation is complete [Condition i)]. We will now use Theorem 5 in conjunction with (17) to prove that we also have a Riesz basis. The maximum eigenvalue can be determined as

$$
\lambda_{\max }\left[\hat{\mathbf{A}}_{\hat{\phi}}\left(e^{j \omega}\right)\right]=\sup _{\|\mathbf{u}\|=1}\left\{\mathbf{u}^{H} \hat{\mathbf{Q}}^{T}\left(e^{-j \omega}\right) \hat{\mathbf{A}}_{\varphi}\left(e^{j \omega}\right) \hat{\mathbf{Q}}\left(e^{j \omega}\right) \mathbf{u}\right\} .
$$

Defining $\mathbf{v}=\hat{\mathbf{Q}}\left(e^{j \omega}\right) \mathbf{u}$, we note that $\|\mathbf{v}\|^{2} \leq \lambda_{\max }\left[\hat{\mathbf{Q}}^{T}\right.$ $\left.\left(e^{-j \omega}\right) \hat{\mathbf{Q}}\left(e^{j \omega}\right)\right]$. Thus

$$
\begin{aligned}
\lambda_{\max }\left[\hat{\mathbf{A}}_{\tilde{\phi}}\left(e^{j \omega}\right)\right] \leq \lambda_{\max }\left[\hat{\mathbf{Q}}^{T}\left(e^{-j \omega}\right) \hat{\mathbf{Q}}\left(e^{j \omega}\right)\right] \\
\cdot \sup _{\|\mathbf{v}\|=1}\left\{\mathbf{v}^{H} \hat{\mathbf{A}}_{\varphi}\left(e^{j \omega}\right) \mathbf{v}\right\} \\
\leq \lambda_{\max }\left[\hat{\mathbf{Q}}^{T}\left(e^{-j \omega}\right) \hat{\mathbf{Q}}\left(e^{j \omega}\right)\right] \\
\cdot \lambda_{\max }\left[\hat{\mathbf{A}}_{\varphi}\left(e^{j \omega}\right)\right] .
\end{aligned}
$$

This allows us to conclude that

$$
\underset{\omega \in[0,2 \pi)}{\operatorname{ess} \sup } \lambda_{\max }\left[\hat{\mathbf{A}}_{\tilde{\phi}}\left(e^{j \omega}\right)\right] \leq M_{Q}^{2} \cdot B_{\varphi}<+\infty
$$


where we use the fact that the constants $B_{\varphi}=$ $\operatorname{ess} \sup _{\omega \in[0,2 \pi)} \lambda_{\max }\left[\hat{\mathbf{A}}_{\varphi}\left(e^{j \omega}\right)\right]=\operatorname{ess}_{\sup } \omega \in[0,2 \pi) \hat{a}_{\varphi}\left(e^{j \omega}\right)$ and $M_{Q}^{2}=\operatorname{ess} \sup _{\omega \in[0,2 \pi)} \lambda_{\max }\left[\hat{\mathbf{Q}}^{T}\left(e^{-j \omega}\right) \hat{\mathbf{Q}}\left(e^{j \omega}\right)\right]$ are both finite by assumption [cf., a2) and a3)]. Similarly, we show that

$$
\underset{\omega \in[0,2 \pi)}{\operatorname{ess} \inf } \lambda_{\min }\left[\hat{\mathbf{A}}_{\tilde{\phi}}\left(e^{j \omega}\right)\right] \geq m_{Q}^{2} \cdot A_{\varphi}>0
$$

where $m_{Q}>0$ and $A_{\varphi}>0$ are the corresponding lower bound constants. Thus, we have established the existence of the constants $A$ and $B$ in Theorem 2.

\section{B. Condition Number in the Orthogonal and Bandlimited Cases}

If the Riesz bounds in a2) are such that $A_{\varphi}=B_{\varphi}=1$, then the basis is orthonormal [2]. In this particular situation, we have $a_{\varphi}(k)=\delta(k)$ (the discrete unit impulse), which implies that $\hat{\mathbf{A}}_{\varphi}(z)$ in (18) is the $m \times m$ identity matrix. Thus, the dual bounds constants in (17) are identical to those that appear in the stability condition a3). An implication is that (16) in the orthogonal case is given by

$$
\alpha_{\text {ortho }}=\frac{M_{Q}}{m_{Q}}
$$

where the constant $M_{Q}$ and $m_{Q}$ are defined in a3). This condition number can therefore be easily evaluated once we have determined the filter matrix $\hat{\mathbf{Q}}(z)$. Note that the invertibility requirement a3) ensures that the reconstruction problem is well posed in the sense that $\alpha_{\text {ortho }}$ is necessarily finite.

One noteworthy example where the basis is orthogonal is the bandlimited case with $\varphi(x)=\operatorname{sinc}(x)$. As we had remarked in our earlier work, this case is best dealt with in the Fourier domain using the so-called modulation representation. Specifically, when $\varphi(x)=\operatorname{sinc}(x)$, we had shown that the modulation matrix is given by (cf., [18, Section V-B])

$$
\begin{aligned}
& \hat{\mathbf{A}}_{\bmod }\left(e^{j \omega}\right)= \\
& {\left[\begin{array}{cccc}
\hat{a}_{1}\left(e^{j \omega}\right) & \hat{a}_{1}\left(e^{j[\omega+2 \pi / m]}\right) & \cdots & \hat{a}_{1}\left(e^{j[\omega+(m-1) 2 \pi / m]}\right) \\
\vdots & \vdots & & \vdots \\
\hat{a}_{m}\left(e^{j \omega}\right) & \hat{a}_{m}\left(e^{j[\omega+2 \pi / m]}\right) & \cdots & \hat{a}_{m}\left(e^{j[\omega+(m-1) 2 \pi / m]}\right)
\end{array}\right]}
\end{aligned}
$$

with

$$
\begin{aligned}
& \hat{a}_{i}\left(e^{j(\omega+2 l \pi / m)}\right)= \\
& \begin{cases}\hat{h}_{i}\left(\omega+\frac{2 l \pi}{m}\right), & -\pi \leq \omega<\pi-\frac{2 l \pi}{m} \\
\hat{h}_{i}\left(\omega+\frac{2 l \pi}{m}-2 \pi\right), & \pi-\frac{2 l \pi}{m} \leq \omega \leq \pi\end{cases}
\end{aligned}
$$

where $\hat{h}_{i}(\omega)$ is the frequency response of the $m$ th analysis filter. Using this modulation matrix, we can obtain another expression for the transfer function of the reconstruction filter $Q$ \{cf., (40) in [18]\}

$$
\hat{\mathbf{Q}}\left(e^{j \omega}\right)=m^{-1 / 2} \cdot \mathbf{D}\left(e^{j(\omega / m)}\right) \cdot \mathbf{G}_{m} \cdot \hat{\mathbf{A}}_{\text {mod }}^{-1}\left(e^{j(\omega / m)}\right)
$$

where $\mathbf{D}\left(e^{j(\omega / m)}\right)=\operatorname{diag}\left(1, e^{j(\omega / m)}, \cdots, e^{j(m-1) \omega / m}\right)$ and where $\mathbf{G}_{m}$ is the $m \times m$ unitary discrete Fourier matrix with entries $\left[\mathbf{G}_{m}\right]_{k, l}=m^{-1 / 2} \cdot e^{j(2 \pi k l / m)}$ with $k, l=$ $0, \cdots, m-1$. Since the matrices $\mathbf{D}\left(e^{j(\omega / m)}\right)$ and $\mathbf{G}_{m}$ in
(26) are unitary, it follows that the singular values of $\hat{\mathbf{Q}}\left(e^{j \omega}\right)$ and $m^{-1 / 2} \cdot \hat{\mathbf{A}}_{\text {mod }}^{-1}\left[e^{j(\omega / m)}\right]$ are identical. In particular, this implies that

$$
\begin{aligned}
& \underset{\omega \in[0,2 \pi)}{\operatorname{essinf}} \lambda_{\min }\left[\hat{\mathbf{A}}_{\text {mod }}^{T}\left(e^{-j \omega}\right) \cdot \hat{\mathbf{A}}_{\text {mod }}\left(e^{j \omega}\right)\right]=\frac{m}{M_{Q}^{2}} \\
& \operatorname{ess} \sup \lambda_{\max }\left[\hat{\mathbf{A}}_{\text {mod }}^{T}\left(e^{-j \omega}\right) \cdot \hat{\mathbf{A}}_{\text {mod }}\left(e^{j \omega}\right)\right]=\frac{m}{m_{Q}^{2}} \\
& \omega \in[0,2 \pi)
\end{aligned}
$$

which gives us an alternative way of computing the bound constants. Note that the argument also holds in the general case where $\varphi(x)$ is arbitrary. Thus, we have effectively shown that the condition number of the system is the same as the condition number of the modulation matrix $\hat{\mathbf{A}}_{\text {mod }}\left(e^{j \omega}\right)$-or the polyphase matrix $\hat{\mathbf{A}}_{\text {poly }}\left(e^{j \omega}\right)=\hat{\mathbf{A}}_{\phi \varphi}\left(e^{j \omega}\right)$ —as long as the basis is orthogonal.

\section{Examples}

To illustrate this aspect of the theory, we will determine these stability measures for various instances of interlaced and derivative sampling. We will consider two alternative methods of reconstruction. The first uses the cubic spline model with $\varphi(x)=\beta^{3}(x)$, where $\beta^{3}$ is the centered cubic B-spline (cf., [18]). The second uses the more standard bandlimited model with $\varphi(x)=\operatorname{sinc}(x)$ (cf., [9], [10]).

1) Interlaced Sampling: In this example with $m=2$, we are sampling the signal $f(x)$ at half the reconstruction rate collecting not one but two samples separated by a distance of $\Delta t$. The corresponding analysis functions are $\phi_{1}(x)=\delta(x)$ and $\phi_{2}(x)=\delta(x-\Delta t)$.

Once we have specified $\varphi$, we can determine the deconvolution filter $\hat{\mathbf{Q}}(z)$ and then use (17) to compute the Riesz bounds $\left(A_{\tilde{\phi}}, B_{\tilde{\phi}}\right)$, which are the quantities of interest. For the two cubic spline examples presented in [18, Fig. 4], we get the values $(0.5773,3.4132)$ for $\Delta t=1 / 2$ and $(0.4857,1)$ for $\Delta t=1$. This is a clear indication that the conditioning of the system is better in the case of uniform sampling $(\Delta t=1)$. To further investigate the overall stability of the algorithm, we computed the condition number (16) as a function of the interlacing parameter $\Delta t$. The graph in Fig. 2(a) shows that the algorithm has the most favorable behavior around $\Delta t=1$ with a region of relative stability for $0.3 \leq \Delta t \leq 1.7$. Beyond that point, the conditioning of the system deteriorates rapidly. Again, this is not surprising since the system is obviously underdetermined for the limiting cases $\Delta t=0$ or $\Delta t=2$, where the same samples are collected twice. We also note that the performance is symmetric with respect to the origin and the point $\Delta t=1$. For comparison, we have included the graph for the bandlimited reconstruction with $\phi(x)=\operatorname{sinc}(x)$ in Fig. 2(b); in this case, the condition number was determined from the modulation matrix, as described in Section III-B. We observe the same qualitative behavior, which is not overly surprising since it is well known that higher order spline interpolants are very similar to their sinc counterparts [1]. Note that the curve achieves the minimum possible value $\alpha_{\tilde{\phi}}=1$ for $\Delta=1$ (uniform sampling), which is the operating point for Shannon's sampling theory. This minimum value reflects the fact that the conventional sinc synthesis functions are orthogonal. 


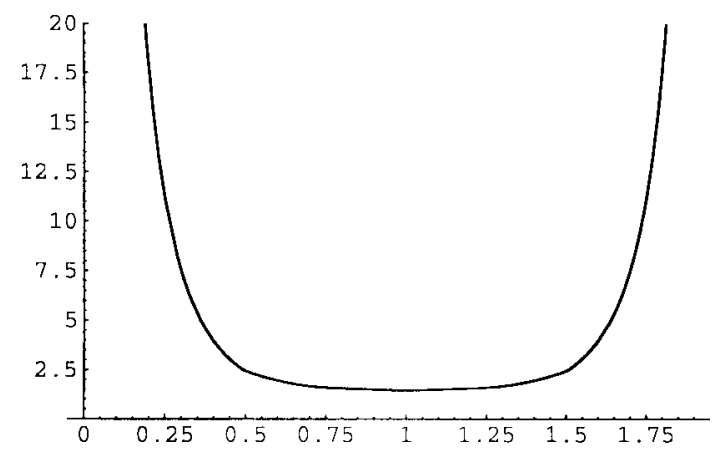

(a)

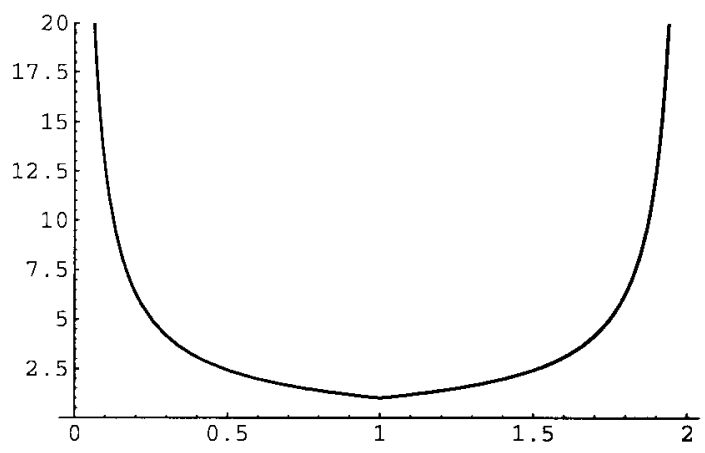

(b)

Fig. 2. Condition number $\alpha_{\tilde{\phi}}$ as a function of the interlacing parameter $\Delta t$. (a) Interlaced sampling with $\varphi(x)=\beta^{3}(x)$ (cubic spline reconstruction). (b) Interlaced sampling with $\varphi(x)=\operatorname{sinc}(x)$ (bandlimited reconstruction).

2) Derivative Sampling: In the next example, we take one sample of the input signal and one sample of its $p$ th derivative with an offset $0 \leq \Delta t \leq 2$. The corresponding analysis functions are $\phi_{1}(x)=\delta(x)$ and $\phi_{2}(x)=\delta^{(p)}(x-\Delta t)$, where $\delta^{(p)}(x)$ denotes the $p$ th derivative of the Dirac-delta function. Fig. 3(a) shows the stability curve for the first derivative sampling using the cubic spline reconstruction. We observe one strong singularity in the center for $\Delta t=1$ and two others for $\Delta t=0,2$, which appear to be specific to splines. The best results are obtained around $\Delta t=0.175,1.825$ with a region of relative stability for $\Delta t \in[0.05,0.7] \cup[1.3,1.95]$. The corresponding graph for the bandlimited case is shown in Fig. 3(b). There is a clear improvement over splines in the boundary regions, but the curve exhibits a somewhat stronger singularity in the center. This result is consistent with the findings of Marks and Cheung, who had already shown the existence of such a point of instability [4], [9]. In general, the situation become less favorable as the order of the derivative increases. In the case of the second derivative (data not shown here), a strong singularity occurs for $\Delta t=0,2$, which again is consistent with the report of Marks for the bandlimited case. For cubic splines, the procedure also diverges for $\Delta=$ $1 / 4,3 / 4$, which is somewhat unexpected. In any case, we do not recommend pushing the derivative sampling procedures too far because of their high sensitivity to noise [8].

\section{PERFormance ANALYSis}

In general, when the input signal $f(x)$ is not in $V(\varphi)$, we cannot achieve a perfect reconstruction because our rep-

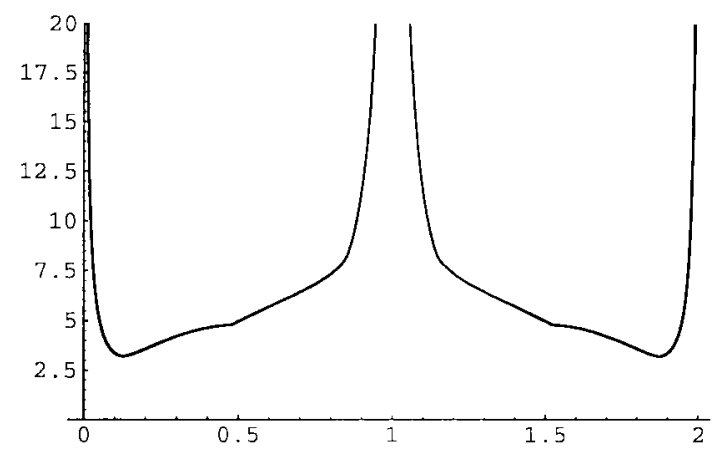

(a)

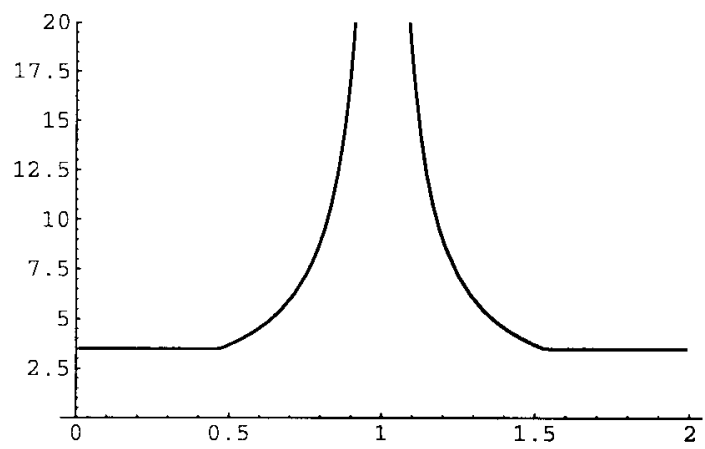

(b)

Fig. 3. Condition number $\alpha_{\tilde{\phi}}$ as a function of the interlacing parameter $\Delta t$. (a) First derivative sampling with $\varphi(x)=\beta^{3}(x)$ (cubic spline reconstruction). (b) First derivative sampling with $\varphi(x)=\operatorname{sinc}(x)$ (bandlimited reconstruction).

resentation subspace is not sufficiently rich. The best possible outcome under those circumstances would be to reconstruct the function in $V(\varphi)$ that is the closest to $f(x)$ in the $L_{2}$ sense (minimum error solution). In this section, we address the crucial issue of how close our approximation $\tilde{f}$ is to this optimal estimate, which is also the orthogonal projection of $f$ into $V(\varphi)$. The question is of importance because the minimum error solution is in general not accessible. However, we would like to have some guarantee that the approximation produced by our system is reasonably close to the best possible estimate.

It turns out that the situation is quite favorable because we are dealing with a projection operator. For this reason, we will first investigate the problem at a more abstract level and then particularize the results for our specific application. We will pay special attention to the case where the $\phi_{i}$ 's are in $L_{2}$ and derive all the relevant bound constants explicitly.

\section{A. Projection Operators: General Properties}

The main results in this subsection are valid for any abstract Hilbert space $\mathcal{H}$. We start with a first inequality that we suspect to be well known to mathematicians.

Proposition 1: Let $V$ be a closed subspace of a Hilbert space $\mathcal{H}$. Then

$\forall f \in \mathcal{H},\|f-P f\| \leq\|f-\tilde{P} f\| \leq \sqrt{1+\|\tilde{P}\|^{2}} \cdot\|f-P f\|$

where $P$ is the orthogonal projector of $\mathcal{H}$ onto $V$, and where $\tilde{P}$ is any projector of $\mathcal{H}$ onto $V$; the constant $\|\tilde{P}\| \geq 1$ denotes the norm of the operator $\tilde{P}$. 
Proof: The closeness assumption is required for the projectors to be well defined. The left part of the inequality is trivial because the orthogonal projector $P$ provides the minimum error approximation. Introducing the identity operator $I$, we write

$$
\|\tilde{P} f-P f\|=\|\tilde{P} \circ(I-P) f\| \leq\|\tilde{P}\| \cdot\|f-P f\|
$$

where we use the fact that $(\tilde{P} \circ P) f=P f(\tilde{P}$ and $P$ are both projectors onto $V)$. Since $(P f-\tilde{P} f) \in V$ and $(f-P f) \in V^{\perp}$, we can write the Pythagorean relation

$$
\|f-\tilde{P} f\|^{2}=\|f-P f\|^{2}+\|\tilde{P} f-P f\|^{2} .
$$

The result then follows by substitution of (30) into (31).

This inequality is directly applicable to our particular situation, provided that we estimate the norm of the operator $\tilde{P}$ in Theorem 1. Specifically, we may use the upper bound

$$
\|\tilde{P}\| \leq C_{\phi} \cdot \sqrt{B_{\tilde{\phi}}}
$$

which is obtained by combining $a 1^{\prime}$ ) with the Riesz bound in Theorem 2. This leads to the error estimate

$$
\|f-\tilde{P} f\| \leq \sqrt{1+C_{\phi}^{2} B_{\tilde{\phi}}} \cdot\|f-P f\|
$$

which shows that the approximation error has the same qualitative behavior as in the orthogonal case. In particular, it implies that both projectors have the same type of $L_{2}$ convergence properties. The error bound (33) is not very sharp, but it is applicable under the most general circumstances [i.e., under hypotheses $\mathrm{a} 1^{\prime}$ ), a2), and a3)]. It is primarily useful when the analysis functions are not in $L_{2}$. This is true, in particular, for all the examples treated in Section III-C.

There are also many other situations of practical interest, which lend themselves to a more precise performance analysis. To sharpen our error bound, we need to get more specific in characterizing the projector. For this purpose, we now consider two subspaces $U$ and $V$ of $\mathcal{H}$ and investigate the projector of $\mathcal{H}$ onto $V$ perpendicular to $U$. This operator, which we denote by $P_{V \perp U}$, is defined as

$$
\begin{aligned}
\forall f_{1} \in V, \quad P_{V \perp U} f_{1} & =f_{1} \\
\forall f_{2} \in U^{\perp}, \quad P_{V \perp U} f_{2} & =0 \\
\forall f \in \mathcal{H}, \quad\left(I-P_{V \perp U}\right) f & \in U^{\perp}
\end{aligned}
$$

where $U^{\perp}$ is the perpendicular complement of $U$ with respect to $\mathcal{H}$. For this particular geometry, we have the following improved bound, the proof of which can be found in [15].

Theorem 3 [Unser-Aldroubi]: Let $U$ and $V$ be two closed subspace of a Hilbert space $\mathcal{H}$ such that $\tilde{P}=P_{V \perp U}$, which is the projector of $\mathcal{H}$ onto $V$ perpendicular to $U$, is well defined. Then

$$
\forall f \in \mathcal{H},\|f-P f\| \leq\|f-\tilde{P} f\| \leq \frac{1}{\cos \left(\theta_{U V}\right)}\|f-P f\|
$$

where $P=P_{V}$ is the orthogonal projector of $\mathcal{H}$ onto $V$. The quantity $\cos \theta_{U V}$ represents the cosine of the "angle" between the subspaces $U$ and $V$ defined by

$$
\cos \left(\theta_{U V}\right)=\inf _{u \in U,\|u\|=1}\left\|P_{V} u\right\| \leq 1 .
$$

In Section IV-C, we will give an explicit formula for $\cos \left(\theta_{U V}\right)$, which will make this error bound directly applicable to the case $\mathcal{H}=L_{2}$. We now end this section by establishing the connection between this inequality and the first error bound in Proposition 1.

Theorem 4: Let $U$ and $V$ be two closed subspaces of $\mathcal{H}$ such that is $P_{V \perp U}$ is well defined. Then

$$
\forall f \in \mathcal{H}, \quad\left\|P_{V \perp U} f\right\| \leq \frac{1}{\cos \left(\theta_{V U}\right)} \cdot\|f\|
$$

where $\cos \left(\theta_{V U}\right)$ is obtained by interchanging $U$ and $V$ in (38).

This last result gives the norm of the projection operator $\left\|P_{V \perp U}\right\|=\|\tilde{P}\|=1 / \cos \left(\theta_{V U}\right)$, which can be directly substituted in the error bound in Proposition 1. In the present situation, it turns out that the "angles" between the spaces $U$ and $V$ are symmetrical, i.e., $\cos \left(\theta_{U V}\right)=\cos \left(\theta_{V U}\right)$ (cf., Theorem 6). It is thus clear that the inequality in Theorem 3 is sharper than the one in Proposition 1, which may differ by as much as a factor of $\sqrt{2}$. However, we should note the "angles," as they have been defined here, are in general not symmetrical for arbitrary spaces $U$ and $V$.

\section{B. Proof of Theorem 4}

Our proof relies on the following identities.

Lemma 1: Let $U$ and $V$ be two closed subspaces of $\mathcal{H}$ such that is $P_{V \perp U}$ well defined. Then, we have the identities

$$
\begin{array}{ll}
\forall f \in \mathcal{H}, & \left(P_{V \perp U} \circ P_{U}\right) f=P_{V \perp U} f \\
\forall f \in \mathcal{H}, & \left(P_{U} \circ P_{V \perp U}\right) f=P_{U} f .
\end{array}
$$

Proof of Lemma 1: Using the projection theorem, we write $f=f_{1}+f_{1}^{\perp}$, where $f_{1}=P_{U} f \in U$, and $f_{1}^{\perp}=\left(I-P_{U}\right) f \in$ $U^{\perp}$. Therefore

$$
P_{V \perp U} f=P_{V \perp U} f_{1}+\underbrace{P_{V \perp U} f_{1}^{\perp}}_{=0}=\left(P_{V \perp U} \circ P_{U}\right) f .
$$

where $P_{V \perp U} f_{1}^{\perp}$ vanishes because of (35). This proves the first identity. A direct consequence of this result is

$$
\begin{aligned}
\forall f \in \mathcal{H}, \quad\left(P_{U} \circ P_{V \perp U}\right) f & =\left(P_{U} \circ P_{V \perp U} \circ P_{U}\right) f \\
& =\left(P_{U} \circ P_{V \perp U}\right) f_{1} .
\end{aligned}
$$

Next, using the decomposition $f_{1}=f_{2}+f_{21}^{\perp}$, where $f_{2}=$ $P_{V \perp U} f_{1} \in V$ and $f_{21}^{\perp}=\left(I-P_{V \perp U}\right) f_{1} \in U^{\perp}$ [cf., (36)], we show that $P_{V \perp U} f_{1}=f_{2}=f_{1}-f_{21}^{\perp}$. This implies that

$$
\forall f_{1} \in U, \quad\left(P_{U} \circ P_{V \perp U}\right) f_{1}=P_{U} f_{1}-\underbrace{P_{U} f_{21}^{\perp}}_{=0}=f_{1} .
$$

The identity (40) then follows from the combination of (41) and (42).

Proof of Theorem 4: Using identity (40) in Lemma 1 and the fact that $\left\|P_{U}\right\|=1$, we have

$$
\forall f \in \mathcal{H}, \quad\left\|P_{U} f_{2}\right\|=\left\|P_{U} f\right\| \leq\|f\|
$$

where $f_{2}=P_{V \perp U} f$. Using the definition of $\cos \left(\theta_{V U}\right)$, we write

$$
\forall f_{2} \in V, \quad \cos \left(\theta_{V U}\right) \cdot\left\|f_{2}\right\| \leq\left\|P_{U} f_{2}\right\| .
$$

We get the desired result by combining both of these inequalities. 


\section{Hilbert Space Analysis in $L_{2}$}

In the particular case where the $\phi_{i}{ }^{\prime}$ 's are in $L_{2}$, we can select $\mathcal{H}=L_{2}$ and construct the complementary subspace $U(\phi)=\operatorname{span}\{\boldsymbol{\Phi}(x-m k)\}_{k \in Z}$ such that $\tilde{P}=P_{V \perp U}$. The reconstruction is performed in the subspace $V=V(\varphi)$, as specified in Section II. This will allow us to determine the quantities $C_{\phi}$ and $\cos \left(\theta_{U V}\right)$ explicitly, as well as to make use of the second improved error bound from the previous section. This analysis relies heavily on the theoretical results of Aldroubi in [3], who considers projectors onto general atomic spaces of the form $U(\phi)=$ span $\left\{O^{k} \boldsymbol{\Phi}(x)\right\}_{k \in Z}$, where $O^{k}$ denotes the $k$ th power of a unitary operator $O: L_{2}\left(R^{m}\right) \rightarrow$ $L_{2}\left(R^{m}\right)$. Here, we will particularize some of his results for the unitary $m$-shift operator $S_{m} \boldsymbol{\Phi}(x)=\boldsymbol{\Phi}(x-m k)$.

We start by giving the appropriate version of [3, Theorem $2.2]$, which gives the necessary and sufficient condition for $U(\phi)$ to be a closed subspace of $L_{2}$.

Theorem 5 [Aldroubi]: The subspace $U(\phi)=\operatorname{span}\{\boldsymbol{\Phi}(x-$ $m k)\}_{k \in Z}$ is a closed subspace of $L_{2}$ with $\{\boldsymbol{\Phi}(x-m k)\}_{k \in Z}$ as its Riesz basis if and only if

Condition a4):

$$
\left\{\begin{array}{l}
A_{\phi}=\underset{\omega \in[0,2 \pi)}{\operatorname{ess} \inf _{\min }\left[\hat{\mathbf{A}}_{\phi}\left(e^{j \omega}\right)\right]>0} \\
B_{\phi}=\underset{\omega \in[0,2 \pi)}{\operatorname{ess} \sup _{\max }\left[\hat{\mathbf{A}}_{\phi}\left(e^{j \omega}\right)\right]<+\infty}
\end{array}\right.
$$

where the $m \times m$ matrix $\hat{\mathbf{A}}_{\phi}(z)$ is the $z$-transform of the autocorrelation matrix sequence $\mathbf{A}_{\phi}(k)=\left\langle\boldsymbol{\Phi}(x-m k), \boldsymbol{\Phi}^{T}(x)\right\rangle$. The two constants $A_{\phi}$ and $B_{\phi}$ are the corresponding lower and upper Riesz bounds.

Next, we show that this leads to a simple way of computing the constant $C_{\phi}$ in $\mathrm{a}^{\prime}$ ).

Proposition 2: Under Assumption a4), there always exists a constant $C_{\phi}$ such that

$$
\forall f \in L_{2}, \quad\left\|\mathbf{g}_{\mathbf{m}}\right\|_{l_{2}} \leq C_{\phi} \cdot\|f\| .
$$

The smallest constant is $C_{\phi}=\sqrt{B_{\phi}}$ where $B_{\phi}$ is the upper Riesz bound for the basis $\{\boldsymbol{\Phi}(x-m k)\}_{k \in Z}$.

In other words, our new Assumption a4) implies a1'), which in turn implies a1). Note that condition a4) rules out the examples in Section III-C, which used Dirac analysis distributions. This limitation can be dealt with easily by adding some form of prefiltering prior to sampling. As will be illustrated shortly in Section IV-D, this may even have some advantages from the point of view of performance; it also corresponds to a more realistic modelization of a physical system.

Proof of Proposition 2: Let $P_{U}$ denote the orthogonal projection of $L_{2}$ onto $U(\phi) . P_{U}$ is a nonexpansive mapping in the sense that $\forall f \in L_{2},\|f\| \geq\left\|P_{U} f\right\|$. The orthogonal projection of $f$ onto $U$ can be written as

$$
\begin{aligned}
P_{U} f(x) & =\sum_{i=1}^{m} \sum_{k \in Z}\left\langle f(x), \phi_{i}(x-m k)\right\rangle \dot{\phi}_{i}(x-m k) \\
& =\sum_{i=1}^{m} \sum_{k \in Z} g_{i}(m k) \stackrel{\circ}{\phi_{i}}(x-m k)
\end{aligned}
$$

which is the representation that uses the dual basis functions of the $\phi_{i}$ 's in $U$. These duals $\dot{\phi}_{i} \in U$, which are not to be confused with the functions $\tilde{\phi}_{i} \in V$ in Theorem 1, are generated by the function-vector $\stackrel{\circ}{\mathbf{\Phi}}(x)=\sum_{k \in Z} \mathbf{A}_{\phi}^{i n v}(k) \boldsymbol{\Phi}(x-k)$, where $\mathbf{A}_{\phi}^{i n v}(k)$ denotes the convolution inverse of $\mathbf{A}_{\phi}(k)$. It is not difficult to show that the dual Riesz bounds are the reciprocal of the usual ones. Thus, by applying the Riesz inequality, we get

$$
\|f\|^{2} \geq\left\|P_{U} f\right\|^{2} \geq \underset{\phi}{A_{\circ}} \cdot\|\mathbf{g}\|_{l_{2}}^{2}=\frac{1}{B_{\phi}} \cdot\|\mathbf{g}\|_{l_{2}}^{2}
$$

which yields the desired result.

Finally, we can use [3, Theorem 3.2] to compute the "angle" between the two subspaces $U(\phi)$ and $V(\varphi)$. In order to state this result, we first define the Fourier matrix

$$
\hat{\mathbf{R}}_{U V}\left(e^{j \omega}\right)=\hat{\mathbf{A}}_{\phi}^{-1 / 2}\left(e^{j \omega}\right) \cdot \hat{\mathbf{A}}_{\phi \varphi}\left(e^{j \omega}\right) \cdot \hat{\mathbf{A}}_{\varphi}^{-1 / 2}\left(e^{j \omega}\right) .
$$

Theorem 6 [Aldroubi]: If the subspaces $U=V(\boldsymbol{\Phi})$ and $V=V(\varphi)$ are such that Conditions a2) and a4) are satisfied, then i) $\cos \left(\theta_{U V}\right)=\cos \left(\theta_{V U}\right)=$ $\sqrt{\operatorname{ess} \inf _{\omega \in[0,2 \pi)} \lambda_{\min }\left[\hat{\mathbf{R}}_{U V}^{T}\left(e^{-j \omega}\right) \cdot \hat{\mathbf{R}}_{U V}\left(e^{j \omega}\right)\right]}, \quad$ and ii) the oblique projector $\tilde{P}=P_{V \perp U}$ is well-defined if and only if $\cos \left(\theta_{U V}\right) \neq 0$.

Note that we have made two minor corrections to the result reported in [3]. The first is the square root on the right of equation i) and the second the definition of the cross-correlation matrix $\mathbf{A}_{\phi \varphi}(k)=\left\langle\boldsymbol{\Phi}(x-m k), \mathbf{\Psi}^{T}(x)\right\rangle$, which is the time-reversed version of that given by Aldroubi. Interestingly, the matrix $\hat{\mathbf{R}}_{U V}\left(e^{j \omega}\right)$ is invariant to the choice of a particular basis for $U$ or $V$. If, instead of the $\varphi$ 's, we use a representation in $V$ in term of the dual functions in Theorem 2 , we get

$$
\hat{\mathbf{R}}_{U V}\left(e^{j \omega}\right)=\hat{\mathbf{A}}_{\phi}^{-1 / 2}\left(e^{j \omega}\right) \cdot \hat{\mathbf{A}}_{\tilde{\phi}}^{-1 / 2}\left(e^{j \omega}\right)
$$

because the $\phi_{i}$ 's and $\tilde{\phi}_{i}$ 's are biorthogonal $\{$ cf., [18, eq. (18)]\}. By using the same technique as for the proof of Theorem 2, we can estimate $\cos \left(\theta_{U V}\right)$ as

$$
\underset{\omega \in[0,2 \pi)}{\operatorname{ess} \inf _{\max }\left[\hat{\mathbf{A}}_{\phi}\left(e^{j \omega}\right)\right] \cdot \lambda_{\max }\left[\hat{\mathbf{A}}_{\tilde{\phi}}\left(e^{j \omega}\right)\right]} \leq \cos \left(\theta_{U V}\right)^{2} \leq 1 .
$$

This yields the series of upper bounds

$$
\frac{1}{\cos \left(\theta_{U V}\right)} \leq \sqrt{B_{\tilde{\phi}} \cdot B_{\phi}} \leq C_{\phi} \cdot M_{Q} \cdot \sqrt{B_{\varphi}}
$$

where the right-hand side follows from (23) in the proof of Theorem 2. Interestingly, we observe that the first inequality is perfectly compatible with the upper bound for $\left\|P_{V \perp U}\right\|=\|\tilde{P}\|$ given by (32). Specifically, Theorem 4 relates $\cos \left(\theta_{U V}\right)=$ $\cos \left(\theta_{V U}\right)$ to the norm of the operator $P_{V \perp U}=\tilde{P}$, whereas Proposition 2 provides the appropriate relation between $C_{\phi}$ and $B_{\phi}$. The main virtue of the upper bound on the right is to make the connection with our assumptions more explicit. This relation clearly shows that a2)-a4) are sufficient for the projector $\tilde{P}$ to be well defined (i.e., bounded) in $L_{2}$. We have already seen that a4) implies a1 ${ }^{\prime}$ ) (cf., Proposition 2). 


\section{Example: Interlaced Sampling with Prefiltering}

To illustrate these concepts, we consider a modification of the interlaced sampling scheme in Section III-C, where the signal is prefiltered prior to sampling. We will use one of the simplest prefilter, namely, the box function or Bspline of degree 0 . This situation corresponds to the choice $\phi_{1}(x)=\beta^{0}(x)$ and $\phi_{2}(x)=\phi_{1}(x-\Delta t)$. As before, we perform the reconstruction in the space of cubic splines with $\varphi(x)=\beta^{3}(x)$. We can easily derive the corresponding crosscorrelation matrix by using the fact that $\left(h_{1} * \varphi\right)(x)=$ $\left(\phi_{1} * \varphi\right)(x)=\beta^{4}(x)$, where $\beta^{4}$ is the $\mathrm{B}$-spline of degree 4. Specifically, for $\Delta t=1 / 2$, we find that

$$
\hat{\mathbf{A}}_{\phi \varphi}(z)=\frac{1}{384}\left[\begin{array}{cc}
z^{-1}+230 & 76 z^{-1}+76 \\
176+16 z & 16 z^{-1}+176
\end{array}\right]
$$

which yields the bound constants $m_{Q}=0.9945$ and $M_{Q}=$ 9.6525. Similarly, using a4), a2), and (17), we compute the corresponding Riesz constants $\left(A_{\phi}, B_{\phi}\right)=(1 / 2,3 / 2),\left(A_{\varphi}\right.$, $\left.B_{\varphi}\right)=(0.05397,1)$, and $\left(A_{\tilde{\phi}}, B_{\tilde{\phi}}\right)=(0.7228,6.1672)$. Next, we apply Theorem 6 to compute $\cos \left(\theta_{U V}\right)=0.543544$. Finally, we can plug-in these numbers in inequality (50), which yields $\|\tilde{P}\|=1.83978 \leq 3.415 \leq 11.822$. Based on Theorem 3, we therefore conclude that the approximation error is within a factor 1.84 of the minimum error solution (orthogonal projection). This turns out to be a quite favorable result; in fact, much better than what we would expect from a simple interpolation without prefiltering. For instance, the asymptotic analysis in [17] shows that a uniform cubic spline interpolation (the best case of interlaced sampling in Section III-C) has a bias of $7.7 \mathrm{~dB}$ with respect to the least square solution. Thus, with prefiltering we can do significantly better, even when the sampling is nonuniform.

We also investigated the performance of this system as a function the interlacing parameter $\Delta t$. The results are shown in Fig. 4, which provides the graphs of $\cos \left(\theta_{U V}\right)$ and $\|\tilde{P}\|=$ $1 / \cos \left(\theta_{U V}\right)$ as a function of $\Delta t$. The second curve has a characteristic U-shape, indicating that we get the best performance (closest to the least squares solution) in the central region around $\Delta t=1$. This graph also suggests that the procedure is ill posed (e.g., $\|\tilde{P}\| \rightarrow+\infty$ ) for $\Delta t=0,2$, which is to be expected since the system is underdetermined (the same sample is collected twice). For the uniform sampling case $\Delta t=1$, we have $1 / \cos \left(\theta_{U V}\right)=1.195$, which is a value that is in agreement with the results for $m=1$ in [15]. Surprisingly enough, this is not the most favorable case. A closer examination of our data reveals [cf., Fig. 4(a)] that the maximum value of $\cos \left(\theta_{U V}\right)$ occurs at a position that is somewhat shifted with respect to the center.

Since Theorem 3 is true irrespective of the sampling step $h$, we can also easily adapt the Strang-Fix convergence results for the least squares case [13] to the present situation, using the same technique as for the proof of [14, Theorem 1]. This yields the general error bound

$$
\left\|f-\tilde{f}_{h}\right\| \leq \frac{C_{n}}{\cos \left(\theta_{U V}\right)} \cdot h^{(n+1)} \cdot\left\|f^{(n+1)}\right\|
$$

where $\tilde{f}_{h}$ denotes the spline approximation of $f$ at sampling step $h ; C_{n}$ is a known constant that does not depend on $f$,

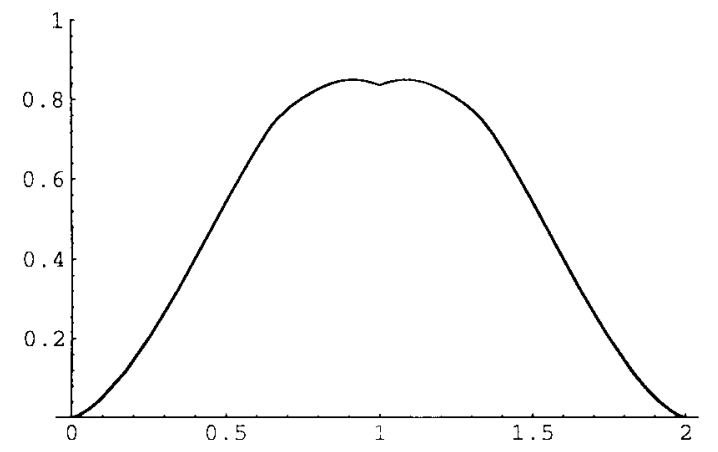

(a)

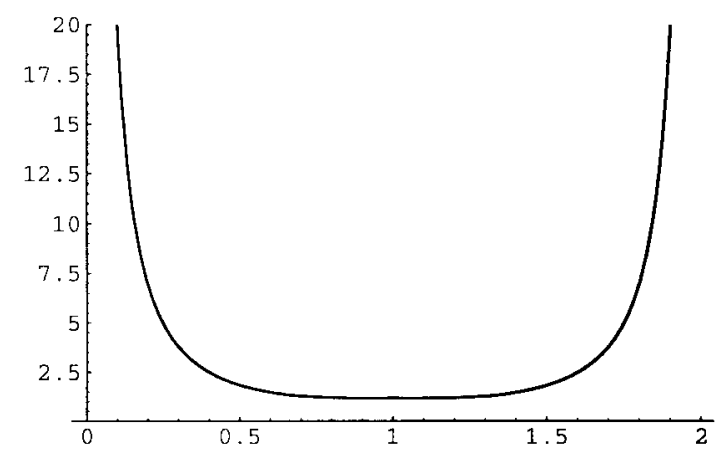

(b)

Fig. 4. Relative performance assessment of the interlaced cubic spline sampling with prefiltering. (a) $\cos \left(\theta_{U V}\right)$ as a function of $\Delta t$. (b) $\|\tilde{P}\|$ as a function of $\Delta t$. The reference is the least squares solution which corresponds to the values $\left\|P_{V}\right\|=1$.

$n=3$ is the degree of the spline, and $\left\|f^{(n+1)}\right\|$ denotes the norm of the $(n+1)$ th derivative of $f$. In other words, the error exhibits an $O\left(h^{n+1}\right)$ decay that is characteristic of spline approximations [17], irrespective of the value of $\Delta t$.

\section{CONCLUSION}

In this paper, we investigated two important aspects of generalized sampling. First, we looked at the stability of the algorithm, which also determines the robustness of the system to noise. The underlying question is: How does a small perturbation of the input (measurements) affect the output of the system? Note that this problem is not entirely conventional because of the type of mismatch between the input of the algorithm, which is discrete, and the system's output, which is continuous. What we have proposed here is to characterize the stability behavior of the algorithm in terms of a condition number. Specifically, we have shown that this condition number can be computed from the Riesz bounds of the dual generating functions in Theorem 1. We have also derived exact formulas for these quantities; this involves searching for the maximum and minimum eigenvalues of some Fourier matrices that characterize the system. The condition number can be used for identifying parameter ranges over which the procedure is unstable (cf., the example of derivative sampling). It is also potentially useful for optimizing acquisition parameters.

Second, we investigated the issue of performance. This is a problem that arises when one attempts to reconstruct an unknown input function $f(x) \in \mathcal{H}$ that is not included in the 
reconstruction subspace $V(\varphi)$. Under those circumstances, the reconstruction cannot be exact, and the best possible outcome is to recover the minimum error approximation of $f, P f$, where $P$ denotes the orthogonal projection operator from $\mathcal{H}$ onto $V(\varphi)$. Although it is generally not possible to reconstruct Pf exactly, we have derived two general $L_{2}$ error bounds that indicate that our generalized sampling solution $\tilde{f}=\tilde{P} f$ is essentially equivalent to the optimal least squares estimate. Specifically, we have shown that $\forall f \in \mathcal{H},\|f-\tilde{P} f\| \leq$ $C_{\phi \varphi} \cdot\|f-P f\|$, where $C_{\phi \varphi} \geq 1$ is a constant that we have determined explicitly.

\section{REFERENCES}

[1] A. Aldroubi, M. Unser, and M. Eden, "Cardinal spline filters: Stability and convergence to the ideal sinc interpolator," Signal Process., vol. 28 , no. 2, pp. 127-138, 1992.

[2] A. Aldroubi and M. Unser, "Sampling procedures in function spaces and asymptotic equivalence with Shannon's sampling theory," Numerical Functional Anal. Optimization, vol. 15, no. 1-2, pp. 1-21, 1994.

[3] A. Aldroubi, "Oblique projections in atomic spaces," Proc. Amer. Math. Soc., vol. 124, no. 7, pp. 2051-2060, 1996.

[4] K. F. Cheung and R. J. Marks, II, "Ill-posed sampling theorems," IEEE Trans. Circuits Syst., vol. CAS-32, pp. 481-484, 1985.

[5] M. K. Tsatsanis and G. B. Giannakis, "Principal component filter banks for optimal wavelet analysis," IEEE Trans. Signal Processing, vol. 43, pp. 1766-1777, Aug. 1995.

[6] A. J. Jerri, "The Shannon sampling theorem-Its various extensions and applications: A tutorial review," Proc. IEEE, vol. 65, no. pp. 1565-1596, 1977.

[7] S. G. Mallat, "A theory of multiresolution signal decomposition: The wavelet representation," IEEE Trans. Pattern Anal. Machine Intell., vol. 11, no. 7, pp. 674-693, July 1989.

[8] R. J. Marks, II, "Noise sensitivity of band-limited signal derivative interpolation," IEEE Trans. Acoust. Speech., Signal Processing, vol. 31, pp. 1028-1032, 1983.

[9] _ Introduction to Shannon Sampling Theory. New York: Springer-Verlag, 1991

[10] A. Papoulis, "Generalized sampling expansion," IEEE Trans. Circuits Syst., vol. CAS-24, pp. 652-654, Nov. 1977.

[11] I. J. Schoenberg, Cardinal Spline Interpolation. Philadelphia, PA: Soc. Ind. Applied Math, 1973.

[12] C. E. Shannon, "Communication in the presence of noise," Proc. IRE, vol. 37, pp. 10-21, 1949.

[13] G. Strang and G. Fix, "A Fourier analysis of the finite element variational method," in Constructive Aspect of Functional Analysis. Rome, Italy: Edizioni Cremonese, 1971, pp. 796-830.

[14] M. Unser, "Approximation power of biorthogonal wavelet expansions," IEEE Trans. Signal Processing, vol. 44, pp. 519-527, Mar. 1995.

[15] M. Unser and A. Aldroubi, "A general sampling theory for nonideal acquisition devices," IEEE Trans. Signal Processing, vol. 42, pp. 2915-2925, Nov. 1994

[16] M. Unser, A. Aldroubi, and M. Eden, "Polynomial spline signal approximations: Filter design and asymptotic equivalence with Shannon's sampling theorem," IEEE Trans. Inform. Theory, vol. 38, pp. 95-103, Jan. 1992.

[17] M. Unser and I. Daubechies, "On the approximation power of convolution-based least squares versus interpolation," IEEE Trans. Signal Processing, vol. 45, pp. 1697-1711, July 1997.
[18] M. Unser and J. Zerubia, "A generalized sampling theory without bandlimiting constraints," IEEE Trans. Circuits Syst., to be published.

[19] M. Vetterli and J. Kovacevic, Wavelets and Subband Coding. Englewood Cliffs, NJ: Prentice-Hall, 1995.

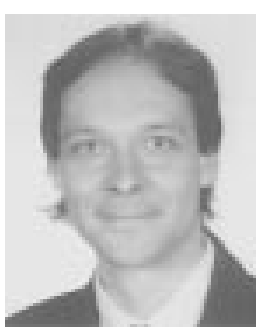

Michael Unser (M'89-SM'94) was born in Zug, Switzerland, on April 9, 1958. He received the M.S (summa cum laude) and Ph.D. degrees in electrical engineering in 1981 and 1984, respectively, from the Swiss Federal Institute of Technology, Lausanne, Switzerland.

From 1985 to 1997, he was with the Biomedical Engineering and Instrumentation Program, National Institutes of Health, Bethesda, MD, where he was head of the Image Processing Group. He is now Professor of Biomedical Image Processing at the Swiss Federal Institute of Technology. His research interests include the application of image processing and pattern recognition techniques to various biomedical problems, multiresolution algorithms, wavelet transforms, and the use of splines in signal processing. He is the author of more than 60 published journal papers in these areas.

Dr. Unser serves as an Associate Editor for the IEEE Signal Processing LETTERS and is a member of the Image and Multidimensional Signal Processing Committee of the IEEE Signal Processing Society. He is also on the editorial boards of Signal Processing and Pattern Recognition and was a former Associate Editor (from 1992 to 1995) for the IEEE TRANSACTIONS on IMAge PRocessing. He co-organized the 1994 IEEE-EMBS Workshop on Wavelets in Medicine and Biology and serves as conference chair for SPIE's Wavelet Applications in Signal and Image Processing, which has been held annually since 1993 . He received the Dommer prize for excellence from the Swiss Federal Institute of Technology in 1981, the research prize of the Brown-Boweri Corporation (Switzerland) for his thesis in 1984, and the IEEE Signal Processing Society's 1995 Best Paper Award (IMDSP technical area) for a paper written with A. Aldroubi and M. Eden on B-spline signal processing.

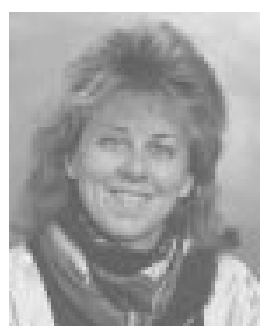

Josiane Zerubia ( $\left.M^{\prime} 88\right)$ received the electrical engineer degree from ENSIEG, Grenoble, France, in 1981, the doctor engineer degree in 1986, the Ph.D. degree in 1988, and the "Habilitation" degree in 1994.

She has been a permanent research scientist at INRIA, Sophia Antipolis, France, since 1989. She had been Director of Research since July 1995 and head of the remote sensing laboratory of PASTIS since November 1995. Previously, she was with the Signal and Image Processing Institute, University of Southern California (USC), Los Angeles, as a post-doctoral researcher. She also worked as a researcher for the University of Nice and CNRS from 1984 to 1988 and in the Research Laboratory of Hewlett Packard in France and in Palo Alto, CA, from 1982 to 1984 . Her current interest is image processing, in particular, image restoration, image segmentation/classification, perceptual grouping, and super-resolution using probabilistic models or neural networks. She also works on parameters estimation and optimization techniques.

Dr. Zerubia is a member of the New York Academy of Sciences since 1996. 